\section{Comparaison du pouvoir pathogène pour la souris d'un stock de Trypanosoma evansi de Mauritanie avec celui de stocks en provenance du Kenya, du Niger, du Tchad et de la Chine}

\author{
M.L. Dia ${ }^{1}$
}

DIA (M.L.). Comparaison du pouvoir pathogène pour la souris d'un stock de Trypanosoma evansi de Mauritanie avec celui de stocks en provenance du Kenya, du Niger, du Tchad et de la Chine. Revue Elev. Méd. vét. Pays trop., 1995, 48 (1) : 21-25

Le pouvoir pathogène d'un stock de Trypanosoma evansi de Mauritanie a été comparé avec celui de stocks en provenance du Kenya, du Niger, du Tchad et de la Chine chez des souris. Les doses inoculées sont de $5.10^{5}$, $2.10^{6}$ el $5.10^{6}$ trypanusumes par suaris selon le lot. Avec T. evansi du Kenya, du Niger et de la Chine, la durée de survie des souris est très courte : en moyenne entre 2,1 et 6,5 jours. En revanche, avec le stock de la Mauritanic ainsi qu'avec celui du Tchad, les souris vivent plus longtemps : entre 12,5 et 22,7 jours selon la dose.

Mots clés : Souris - Trypanosoma evansi - Chine - Kenya - Mauritanie - Niger - Tchad.

\section{Introduction}

Tous les animaux de laboratoire sont sensibles à Trypanosoma evansi. La durée de leur survie varie selon la virulence de la souche (7) et de la concentration de l'inoculum $(1,4,5)$. C'est dans cette optique que l'on a comparé chez la souris, le pouvoir pathogène de $T$. evansi isolé de Mauritanie avec celui de stocks provenant de la Chine, du Kenya, du Niger et du Tchad.

Les critères d'appréciation de ce pouvoir pathogène sont la parasitémie et le temps de survie des souris. Les parasites qui se révèlent peu pathogènes pourront servir à des recherches d'avenir en matière d'essais d'immunisation d'animaux de laboratoire contre $T$. evansi.

\section{Matériel et Méthodes}

Les expériences ont été conduites au laboratoire de Parasitologie de l'Ecole nationale vétérinaire de Lyon. Elles ont duré quatre mois. L'expérience a nécessité 130 souris (65 mâles et 65 femelles). Ce sont des souris blanches (Mus musculus) originaires de l'élevage du laboratoire de l'Ecole nationale vétérinaire de Lyon. Elles sont âgées de 3 semaines et pèsent entre 20 et $30 \mathrm{~g}$ (moyenne 27,12 $\pm 4,5 \mathrm{~g}$ ). Elles sont réparties en lots et placées dans des cages.

1. Centre national d'Elevage et de Recherches vétérinaires, BP 167, Nouakchott, Mauritanie.

Reçu le 5.5.1992, accepté le 28.3.1995.

\section{Stocks}

Cinq stocks sont utilisés. Celui de Chine, isolé d'un buffle dans la province de Zhejang en octobre 1988, a été inoculé à des cobayes transportés en France puis multiplié sur souris à Lyon. Celui du Kenya, (offert par le CRTA de Bobo-Dioulasso, Burkina Faso) a été isolé d'un dromadaire et multiplié sur souris. Celui du Niger, (offert par le CIRAD-IEMVT) a été isolé d'un dromadaire en mars 1988, puis multiplié sur souris en France. Celui de Mauritanie, isolé en juillet 1990 d'un dromadaire femelle dans la région du Trarza, a été inoculé à des lapins transportés en France puis multiplié sur souris à Lyon, et celui du Tchad (offert par le CIRAD-IEMVT) a été isolé d'un dromadaire en juillet 1989, puis multiplié sur souris en France.

Tous ces stocks sont conservés dans les mêmes conditions à Lyon (cryotubes avec citrate de Na, PSG + $10 \mathrm{p}$. 100 de glycérol et stockés à $-80^{\circ} \mathrm{C}$ ). Au moment de l'emploi, ils sont décongelés brutalement sous un robinet d'eau chaude.

\section{Protocole expérimental et dosages}

Après décongélation, les stabilats sont inoculés à des souris qui seront sacrifiées en pic de parasitémie. Le sang de ces souris dilué dans du PBS sera utilisé pour l'expérimentation. Les doses utilisées sont de $5.10^{5}$ (dose D1), $2.10^{6}$ (D2) et $5.10^{6}$ (D3) trypanosomes par souris.

\section{Première expérience}

Trois lots de 6 souris pour chacun des 5 stocks cités sont inoculés à la dose D1 pour le lot 1, la dose D2 pour le lot 2 et la dose D3 pour le lot 3.

\section{Deuxième expérience}

Trois lots de 12 souris sont infectés par $T$. evansi de Mauritanie. Les doses employées sont les mêmes que celles de la première expérience.

\section{Troisième expérience}

Deux lots de 10 souris sont infectés par $T$. evansi de Mauritanie et du Niger à la dose D1 pour le lot 1 et la dose D3 pour le lot 2.

\section{Inoculation et contrôle}

Pour chaque expérience, un lot de 6 souris témoins reçoit $0,1 \mathrm{ml}$ de PBS par souris. Toutes les infections se font par voie intrapéritonéale. La parasitémie est contrôlée quotidiennement à l'aide d'une goutte de sang frais prélevée à la queue et placée entre lame et lamelle. Pour des parasitémies ne dépassant pas 30 trypanosomes par champ, la moyenne de 50 champs a été calculée. Audelà de cette valeur, on procède à une estimation selon la méthode proposée par Herbert et Lumdsen (2). L'échelle retenue est la suivante : 
Communication

Négatif $(\mathrm{N})$; Faible parasitémie $(+):-1 ;(++): 1-10$; $(+++): 10-30 ;(++++): 30-60 ;(+++++):+60$ trypanosomes par champ.

Les résultats des durées de survie sont traités statistiquement par le test d'analyse de variance (F) ou de comparaison de moyenne (t) (9).

\section{Résultats}

\section{Parasitémie}

Vingt-quatre heures après l'infection, on observe :

- à la dose D1, de rares parasites avec $T$, evansi du Kenya. Les souris infectées avec les autres stocks sont négatives ;

- à la dose D2, très peu de parasites (1 à 3 sur toutes les lames) avec T. evansi de Mauritanie et du Tchad. Avec les stocks Kenya, Niger et Chine, la parasitémie des souris varie entre $(+)$ et $(++)$;

- à la dose D3, pour toutes les souris, la parasitémie est intermédiaire : entre $(\mathrm{N})$ et $(++)$.

Quarante-huit heures après l'infection :

- avec T. evansi Kenya, Chine et Niger, la multiplication des parasites est très rapide. Le pic de parasitémie est atteint en 2-3 jours au maximum après la détection des premiers parasites ;

- avec T. evansi de Mauritanie et du Tchad, on observe, tantôt une multiplication progressive des parasites, tantôt des pics de parasitémie suivis de trypanolyses conformes à l'exemple illustré dans la figure 1 .

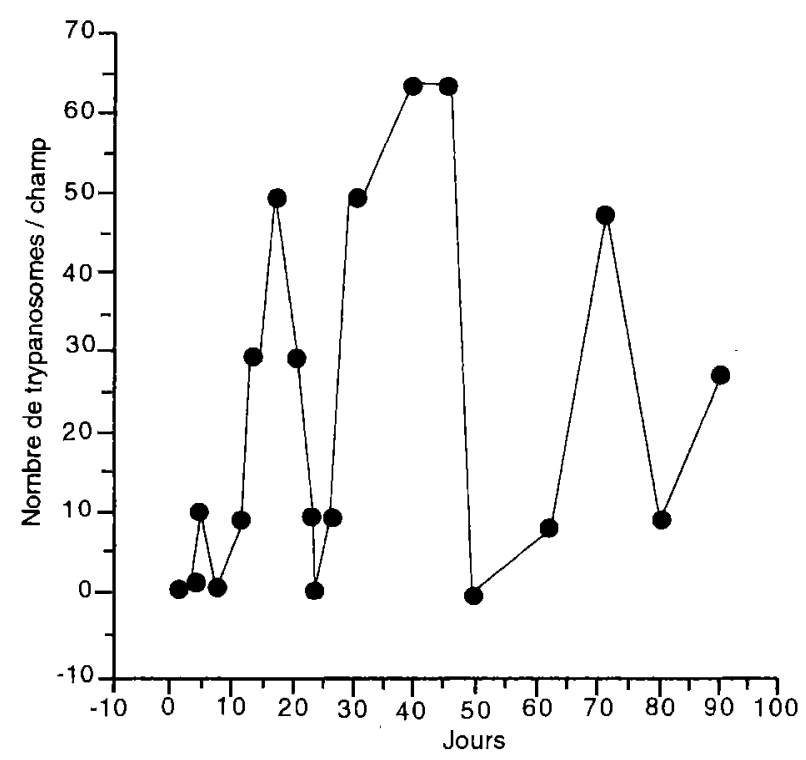

Figure 1 : Evolution de la parasitémie de la souris $n^{\circ} 2$ infectée par $\mathrm{T}$. evansi de Mauritanie (expérience 2). 
TABLEAU I

Durée de survie des souris après infection

(Expérience I)

\begin{tabular}{|c|c|c|c|c|c|c|}
\hline Dose Lot & $\begin{array}{l}\text { Durée survie } \\
\text { (jours) }\end{array}$ & "Niger & Chine & Kenya & Tchad & Mauritanie \\
\hline $\begin{array}{l}5.10^{5} \\
\text { Trypanosomes } \\
\text { par souns. } 1\end{array}$ & $\begin{array}{c}\text { Durée moyenne } \\
\text { Valeurs extrêmes } \\
\text { Ecart-types }\end{array}$ & $\begin{array}{c}4,66 \\
4 \text { et } 5 \\
0,47\end{array}$ & $\begin{array}{l}6,50 \\
6 \text { ett } \\
0,50\end{array}$ & $\begin{array}{c}4 \\
3 \text { et } 5 \\
0,57\end{array}$ & $\begin{array}{c}22,66 \\
16 \text { et } 40 \\
8,11\end{array}$ & $\begin{array}{c}21,88 \\
12 \text { et } 26 \\
4,60\end{array}$ \\
\hline $\begin{array}{l}2.10^{6} \\
\text { Trypanosomes } \\
\text { par souris }\end{array}$ & $\begin{array}{l}\text { Durée moyenne } \\
\text { Valeurs extrêmes } \\
\text { Ecart-types }\end{array}$ & $\begin{array}{l}3,80 \\
3 \text { et } 5 \\
0,68\end{array}$ & $\begin{array}{l}316 \\
2 \text { et } 4 \\
0,68\end{array}$ & $\begin{array}{l}3,33 \\
3 \text { et } 4 \\
0,47\end{array}$ & $\begin{array}{c}17 \\
15 \text { et } 29 \\
6,21\end{array}$ & $\begin{array}{c}21 \\
16 \text { et } 24 \\
2,50\end{array}$ \\
\hline $\begin{array}{l}5.10^{6} \\
\text { Trypanosomes } \\
\text { par souris }\end{array}$ & $\begin{array}{l}\text { Durée moyenne } \\
\text { Valeurs extrêmes } \\
\text { Ecart-types }\end{array}$ & $\begin{array}{c}3,50 \\
3 \text { et } 4 \\
0,50\end{array}$ & $\begin{array}{c}2,16 \\
2, \mathrm{et} 3 \\
0,40\end{array}$ & $\begin{array}{c}3 \\
2 \text { et } 4 \\
0,57\end{array}$ & $\begin{array}{c}12,50 \\
3 \text { et } 39 \\
12,07\end{array}$ & $\begin{array}{c}16,50 \\
12 \text { et } 19 \\
2,62\end{array}$ \\
\hline
\end{tabular}

Tableau II

Durée de survie des souris après infection

(Expérience 2 avec $T$. evansi de Mauritanie)

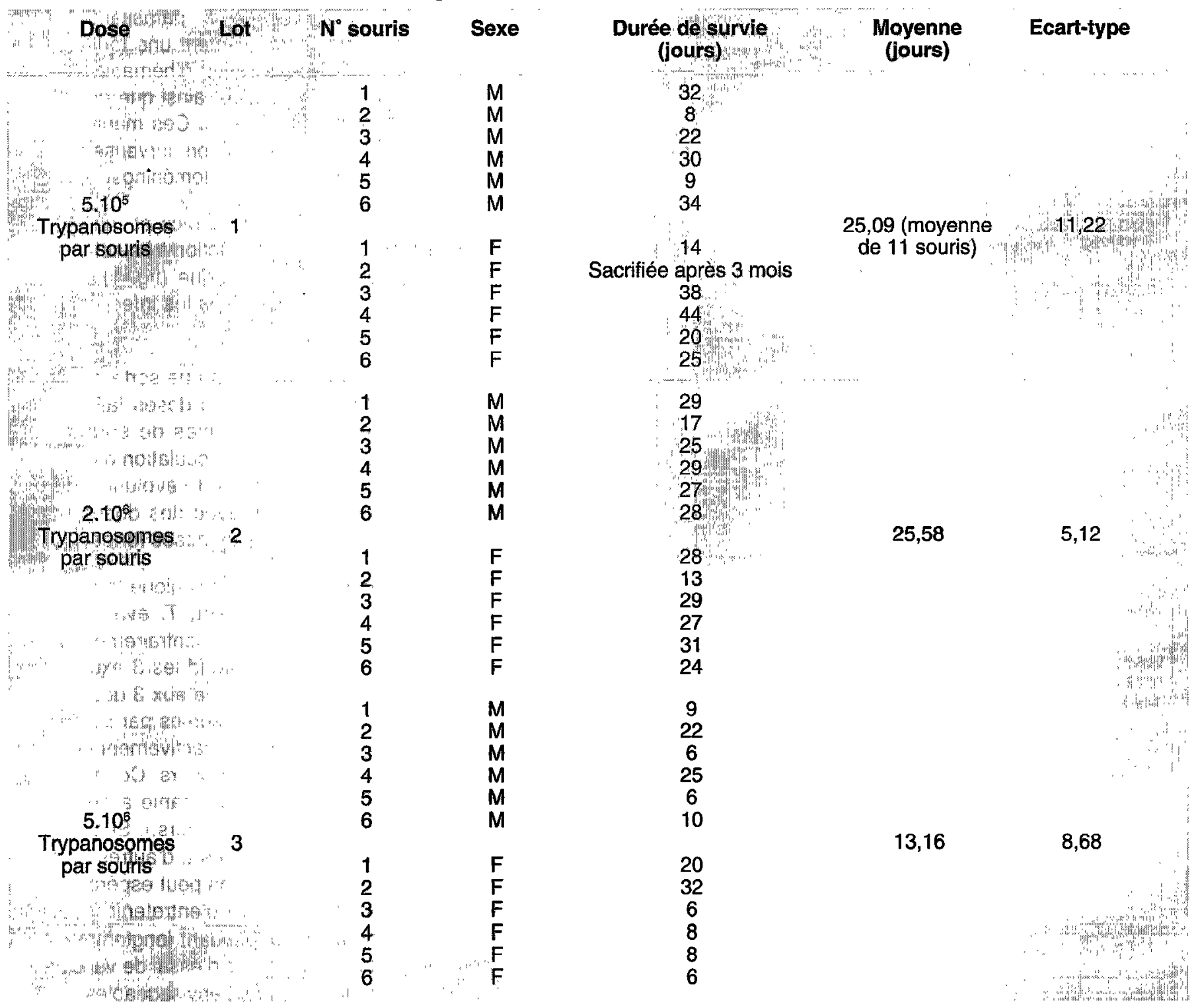


en particulier avec le stock de Chine à la dose D3 $(p<0,001)$.

Groupe 2: $T$. evansi Tchad, les différences ne sont pas significatives. Selon les doses, les valeurs de $t$ varient de 0,31 à $1,06(p>0,05)$.

\section{Effet dose}

Pour les 4 stocks (Kenya, Chine, Niger et même Tchad), il y a un effet dose net. Les différences des moyennes sont significatives $(p<0,05)$.

Avec $T$. evansi de Mauritanie, les différences ne sont pas significatives, mais la valeur de $\mathrm{F}^{2}{ }_{15}$ qui est de 3,25 est très proche de 3,68 , valeur théorique $(p>0,05)$.

\section{Deuxième expérience}

Les résultats de cette expérience (avec $T$. evansi de Mauritanie) sont regroupés dans le tableau II. Une souris du lot 1 inoculée à la dose D1 est restée avec une parasitémie fluctuante pendant 3 mois. Elle a été sacrifiée au jour J 90. Son évolution est représentée dans la figure 1. Pour les autres souris, la durée moyenne de survie est de $25,09 \pm 11,20$ (D1), de $25,58 \pm 5,12$ (D2) et de $13,16 \pm 8,68$ jours (D3). Les différences entre les doses sont significatives $(F=7,19, p<0,001)$.

\section{Troisième expérience}

Les résultats de cette expérience sont consignés dans le tableau III. Avec la souche Niger, la durée moyenne de survie à la D1 est de $4,33 \pm 0,64$ et à la D3 de 3,70 \pm 1,48 jours. Avec celle de la Mauritanie, les valeurs correspondantes sont $19,30 \pm 6,48$ et $11,40 \pm 6,08$ jours.

TABLEAU III

Durée de survie des souris après infection (Expérience 3)

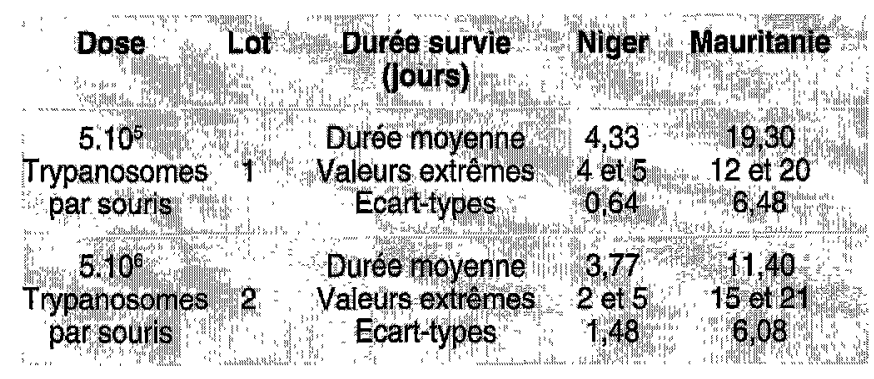

\section{Discussion}

Selon de nombreux auteurs, la capacité de multiplication est l'élément fondamental de la virulence des trypanosomes $(5,7)$. Si l'on entend par capacité de multiplica- tion, une multiplication progressive dans le temps, il faut interpréter ces résultats avec réserve car l'évolution de la parasitémie, surtout à la dose D3, est plus ou moins similaire entre les stocks. Mais si l'on entend par capacité de multiplication, une multiplication exponentielle rapide débordant les défenses de l'hôte, il y a une différence nette entre eux.

Avec $T$. evansi Chine; Kenya et Niger, la multiplication se fait très vite. Le pic de parasitémie est atteint rapidement, la souris meurt en 24-48 $h$ maximum après ce premier pic.

Avec T. evansi de Mauritanie (et Tchad), le comportement est très différent :

- mortalité des souris, 3-8 jours après le premier pic de parasitémie. Durant tout ce temps, la parasitémie est de $(++++)$ à $(+++++)$, les souris présentent une forte anémie (constatée par un nombre très réduit d'hématies dans la goutte de sang prélevée à la queue) ainsi que des signes nerveux (tremblement et tétanisation). Ces manifestations pourraient s'expliquer par la localisation nerveuse du parasite qui aurait franchi la barrière hématoméningée (1) ;

- mortalité des souris après 1 ou 2 pics et même plus, suivis d'absence "totale" ou de réduction très nette de trypanosomes dans le sang périphérique (fig. 1). Ce type d'évolution est souvent rapporté dans les infections naturelles à $T$. evansi $(7,8)$.

Souvent, on assiste avec le temps à une sorte de "rattrapage" des souris infectées avec des doses faibles à tel point qu'on observe des parasitémies de souris identiques malgré des doses initiales d'inoculation différentes. Parfois, dans ces conditions, quand l'évolution dure, il arrive que des souris inoculées avec des doses faibles meurent avant celles ayant reçu des doses fortes.

Si l'on considère comme critère pathologique le temps de survie des souris, indiscutablement, $T$. evansi Chine, Kenya et Niger sont très pathogènes, contrairement à celui de Mauritanie. En effet, si l'on confond les 3 expériences réalisées avec $T$. evansi de Mauritanie aux 3 doses infectantes $\left(5.10^{5}, 2.10^{6}\right.$ et $5.10^{6}$ trypanosomes par souris), les durées de survie moyennes sont respectivement de 22,28 $\pm 8,84,24,05 \pm 4,92$ et $13,25 \pm 7,10$ jours. Ce faible pouvoir pathogène de $T$. evansi de Mauritanie a également été observé par Bourdoiseau (comm. pers.). Si ces résultats chez la souris sont transposables à d'autres animaux de laboratoire (lapin par exemple), on peut espérer que ce faible pouvoir pathogène permette d'entretenir $T$. evansi de Mauritanie sur ces animaux pendant longtemps. Dans ce cas, des perspectives en matière d'essai de vaccination des animaux de laboratoire seraient envisageables. 


\section{Conclusion}

Des souris infectées par $T$. evansi isolé de Mauritanie, du Kenya, de Chine, du Niger et du Tchad ont présenté des parasitémies et des durées de survie variables selon la souche et la concentration de l'inoculum. Les isolats Kenya, Chine et Niger se sont révélés très pathogènes. Ceux de Mauritanie et du Tchad sont peu pathogènes. Toutefois, on sait que la virulence des stocks peut être fortement influencée par des passages mécaniques, au laboratoire, ce qui pourrait expliquer en partie les différences trouvées lors de cette étude.

\section{Remerciements}

L'auteur remercie MM. Bourdoiseau, Dang et Mme Gauthey du laboratoire de Parasitologie de l'Ecole nationale vétérinaire de Lyon pour leur précieuse collaboration.

\section{Bibliographie}

1. BALIS J., 1968. Note sur la répartition de T. evansi dans l'organisme du rat. Revue Elev. Méd. vét. Pays trop., 21 (1) : 101-102.

2. HERBERT W.J., LUMDSEN W.H.R., 1976. Trypanosoma brucei: A rapid "matching" method for estimating the host's parasitemia. Expl Parasit., 28: 521-534.

3. JATKAR P.R., MOHAN SINGH P., 1971. Pathogenesis of anemia in $T$. evansi infection. Part I. Haematology. Indian vet. J., 48: 239-243.

4. JONES T.W., 1985. Antigenic variation in T. evansi: variable antigenic type development in mice, sheep and goats. Trop. Med. Parasit., 36: 53-57.

5. KAGERUKA P., 1982. Contribution à l'étude du sous-genre Trypanozoon et en particulier de la virulence de Trypanosoma (Irypanozoon) evan si (Steel 1885) Balbiani 1888. Thèse Doct., Utrecht, Pays- Bas, 233 p.

6. LUCKINS A.G., McINTYRE N., RAE P.F., 1992. Multiplication of Trypanosoma evansi at the site of infection in skin of rabbits and cattle. Acta trop., 50: 19-27.

7. MAHMOUD M.M., GRAY A.R., 1980. Trypanosomiasis due to T. evansi (Steel 1885) Balbiani 1888. A review of recent research. Trop. Anim. Ilth Prod., 12: 35-47.

8. RÖTTCHER D., SCHILLINGER D., ZWEYGARTH E., 1987. Trypanosomiasis in the camel (Camelus dromedarius). Revue sci. tech. Off. int. Epizoot., 6: 463-470.

9. SWARTZ D, 1991. Méthodes statistiques à l’usage des médecins et des biologistes. Paris, France, Flammarion, 306 p.

DIA (M.L.). Comparison of the pathogenicity to mice of a stock of $T r$ panosoma evansi from Mauritania with that of stocks from Kenya, Niger, Chad and China. Revue Elev. Méd. vét. Pays trop., 1995, 48 (1): 21-25

The pathogenicity to mice of a stock of Trypanosoma evansi from Mauritania has been compared to that of stocks from Kenya, Niger, Chad and China, using doses of $5.10^{5}, 2.10^{6}$ and $5.10^{6}$ trypanosomes per mouse. The survival of mice inoculated with stocks from Kenya, Niger and China is short, on average 2.1 to 6.5 days. Mice inoculated with stocks from Mauritania and Chad survive longer, 12.5 to 22.7 days, depending on the dose.

Key words : Mouse - Trypanosoma evansi - China - Kenya - Mauritania - Niger - Chad

\section{A survey of dermatophilosis in Israeli dairy cattle}

\author{
I. Yeruham ${ }^{1}$ \\ D. $\mathrm{Elad}^{2}$
}

A. Nyska ${ }^{2 *}$

YERUHAM (I.), ELAD (D.), NYSKA (A.). Enquête sur la dermatophilose des bovins laitiers en Israël. Revue Élev. Méd. vét. Pays trop., 1995 48 (1) : 25-29

En Israël la dermatophilose cause de sévères lésions, en particulier chez les vaches laitières durant les premières semaines qui suivent leur primoparturition. Une baisse moyenne de 40 p. 100 de la production du lait a été constatée. Chez ces animaux, on observe une dermatite exsudative aiguë avec une réaction sévère. 580 animaux dans les troupeaux de 38 kibboutz et 15 dans 4 autres petits troupeaux ont ainsi fait l'objet d'une antibiothérapie. Tous les sujets étaient également atteints d'endométrite de métrite ou de mammite. La forme tumorale de la dermatophilose a été observée chez les veaux jusqu'à l'âge de 3 semaines. La dermatophilose était particulièrement visible $(89,4$ p. 100) chez les troupeaux dans lesquels on pratiquait des douches intensives des vaches laitières durant le printemps et l'été, comparativement aux petits troupeaux où la morbidité est plus basse $(6,6$ p. 100). La morbidité était plus élevée dans la plaine côtière méditerranéenne $(66,3$ p. 100) que dans les régions arides ou semi-arides (Negev et vallée de l'Arava) dans le sud d'Israèl (3,7 p. 100). Les auteurs décrivent 5 formes cliniques de la dermatophilose ainsi que les aspects épizootiologiques de la maladie chez les troupeaux israéliens. Pour conclure, une diminution du risque de la maladie peut être obtenue en réduisant la fréquence et l'intensité des douches pratiquées actuellement dans les kibboutz.

Mots clés : Bovin - Bovin laitier - Dermatophilose - Enquête - Epidémiologie - Bactériologie - Histopathologie - Production laitière - Israël.

\section{Introduction}

Dermatophilosis is a contagious skin disease caused by Dermatophilus congolensis. The disease is a non-pruritic disease, characterized by exudative, proliferative or hyperkeratotic dermatitis, accompanied by the production of crusts and folliculitis (14). The causative agent of dermatophilosis is a Gram-positive microorganism belonging to the class Actinomycetales, family Dermatophilaceae, genus Dermatophilus. It is highly resistant to environmental factors (9). The life cycle, morphology and culture characteristics of $D$. congolensis have been described (18). Different clinical forms of dermatophilosis in cattle have been described $(5,7,11,21)$.

1. Hachaklait Gedera and the Koret School of Veterinary Medicine, The Hebrew University of Jerusalem, Bet-Dagan 50250, Israël.

2. The Kimron Veterinary Institute, Bet-Dagan 50250 , and the Kore School of Veterinary Medicine, The Hebrew University of Jerusalem, Bet-Dagan 50250, Israël.

* Adresse actuelle : Department of Pathology, The Kimron Veterinary Institute, POB 12, Bet Dagan 50250, Israël.

Reçu le 15.11.1994, accepté le 26.4.1995. 